\title{
Estudo exploratório para aplicação de modelos de transportes baseados em atividades no Brasil
}

\author{
Fabiana Serra de Arruda; Antônio Nelson Rodrigues da Silva²; Harry Timmermans ${ }^{3}$
}

\begin{abstract}
Resumo: Os modelos baseados em atividades têm sido apontados por diversos pesquisadores como sendo ferramentas promissoras no processo de análise de demanda por transportes. São modelos robustos, que incorporam em sua estrutura uma grande quantidade de variáveis e, dessa forma, produzem resultados mais detalhados sobre o comportamento de viagens individuais. Esses modelos vêm sendo usados para aplicações práticas em várias cidades do mundo. Como no Brasil não existem até o momento registros sistemáticos de sua aplicação, essa pesquisa tem como objetivo apresentar e discutir o potencial e as dificuldades de implementação de um modelo baseado em atividades em uma cidade brasileira de porte médio. Esse processo é composto por três etapas: coleta dos dados, adaptação do modelo às condições brasileiras e análise dos resultados obtidos na fase de calibração. Mesmo sem envolver a fase de validação do modelo, os resultados apontam a viabilidade da aplicação e mesmo o desenvolvimento de modelos de atividades no Brasil.
\end{abstract}

\begin{abstract}
Activity-based models are described by several researchers as promising tools in the process of transportation demand analysis. They are robust models that contain a large number of variables in their structure, allowing them to produce detailed descriptions of individual trip behaviors. As a consequence, these models are being used in practical applications in many cities around the world. However, as we had no information of their systematic application in Brazil hitherto, the main aim of this study is to show and to discuss the potential and the difficulties of implementation of an activity-based model in a Brazilian medium-sized city. The analysis focus on three aspects: data collection, adaptation of the model to the conditions prevailing in Brazilian cities, and analysis of the results of the calibration phase. Notwithstanding the absence of the model validation phase in the study, the results suggest the viability of the application and even of the development of activity-based models in Brazil.
\end{abstract}

\section{INTRODUÇÃO}

Uma das etapas fundamentais no que se refere aos estudos de transportes é o planejamento. Para tanto, planejadores e pesquisadores dispõem de uma grande quantidade de ferramentas que auxiliam no processo de tomada de decisão sobre quais intervenções devem ser analisadas em um determinado estudo e quais as conseqüências destas.

Entre essas ferramentas podem ser citados os modelos de demanda por transportes, dos quais o mais tradicional e conhecido é o modelo de quatro etapas, desenvolvido na década de 1950. Esses modelos, apesar de ainda usados, falham por não incorporar dimensões importantes em sua estrutura, não fornecendo, assim, informações detalhadas sobre o comportamento de viagem. Na década de 1970 surgiram os modelos baseados em atividades, como ferramenta alternativa no processo de análise sobre viagens e atividades realizadas pelos indivíduos.

\footnotetext{
${ }^{1}$ Fabiana Serra de Arruda, Consultora de Transportes. Brasília, DF, Brasil (e-mail: fabiana@tcbr.com.br).

${ }^{2}$ Antônio Nelson Rodrigues da Silva, Escola de Engenharia de São Carlos, Universidade de São Paulo. São Carlos, SP, Brasil (email: anelson@sc.usp.br).

${ }^{3}$ Harry Timmermans, Urban Planning Group, Eindhoven University of Technology. Eidhoven, Netherland (e-mail: h.j.p.timmermans@tue.nl).
} número 2, de dezembro de 2007. ISSN: 1415-7713.
Os modelos de atividades são ferramentas de análise do comportamento de viagem individual que podem ser usados no processo de planejamento de transportes. Eles partem do princípio que a demanda de viagem é derivada da necessidade individual de realização de atividades dispersas no espaço urbano. São modelos mais complexos que os tradicionalmente usados por incorporarem um grande conjunto de opções disponíveis ao indivíduo para programar sua agenda diária de atividades e viagens.

Esses modelos já vêm sendo utilizados em vários países em aplicações práticas (Arentze e Timmermans, 2000), obtendo resultados satisfatórios. No Brasil, no entanto, ainda não existem até o momento registros de aplicação direta e sistemática desses modelos, embora já tenham começado a surgir pesquisas que se baseiam na fundamentação teórica dos modelos baseados em atividades, como no caso de Silva (2006) e Pitombo (2007). Nesse contexto, o objetivo deste artigo é apresentar e discutir o processo de implementação desses modelos nas condições das cidades brasileiras, com ênfase na viabilidade de sua aplicação e nas dificuldades encontradas.

Após essa introdução, o trabalho começa por apresentar, no item 2, uma síntese da literatura que trata dos modelos baseados em atividades, dos quais é destacado um modelo para aplicação em um caso no Brasil. Os detalhes da aplicação são apresentados no item 3 , que trata ainda dos enfoques de avaliação: i) a coleta dos dados necessários à calibração, ii) a sua adapta- 
ção às condições da cidade e iii) a análise dos resultados. Na seqüência são apresentados e discutidos os resultados encontrados no estudo de caso, segundo os três aspectos mencionados no item anterior. No item 5 são apresentadas as conclusões e ao final do artigo são listadas as principais referências bibliográficas.

\section{OS MODELOS BASEADOS EM ATIVIDADES}

Os primeiros trabalhos realizados por Hägerstrand (1970), Chapin (1971) e Cullen e Godson (1975) formam a base das pesquisas sobre análise de atividades. Chapin (1971) propôs uma estrutura na qual as restrições sociais e as motivações inerentes ao indivíduo interagem para moldar os padrões de comportamento de realização de atividades. Hägerstrand (1970), por outro lado, enfatizou as restrições impostas a um indivíduo para sua participação em atividades dispersas no tempo e no espaço, formalizando o conceito de prisma espaço-tempo. Um prisma espaço-tempo pode ser utilizado para definir quais regiões de tempo e espaço podem ser alcançadas por um indivíduo, dado um conjunto de restrições a ele imposto (restrições de capacidade, restrições de acoplamento e restrições de autoridade) e o modo de transporte utilizado. Ainda nos dias de hoje, pesquisas vêm sendo realizadas com o intuito de identificar qual o conjunto de variáveis que efetivamente podem afetar as decisões individuais sobre a distribuição de suas atividades no espaço e no tempo, além daquelas tradicionalmente conhecidas, ou seja, características domiciliares e individuais (Joh e Huh, 2005).

Um dos princípios básicos dos modelos baseados em atividades é que a demanda por transporte é derivada da necessidade que as pessoas têm em realizar suas atividades diárias distribuídas no tempo e no espaço. Esses modelos procuram simular como os indivíduos em um domicílio organizam suas programações de atividades diárias no tempo e no espaço e, a partir daí, avaliam quais as viagens necessárias para que esta programação de atividades possa ser cumprida.

Esta abordagem reconhece as complexas interações entre a participação em atividades e o comportamento de viagem individual. Assim sendo, as atividades geradoras da demanda de transportes são o foco para o entendimento do comportamento de viagem individual, dentro das quais as relações de causa e efeito que determinam as decisões de viagem podem ser modeladas (Pendyala et al., 1998). Entender como as pessoas organizam suas atividades diárias no tempo e no espaço permite aos pesquisadores desenvolver modelos que possam prever as posições mais indicadas em que atividades não planejadas podem ser inseridas em programações já estabelecidas, durante um processo contínuo de programação (Mohammadian e Doherty, 2005). Esses modelos são ferramentas que verificam os mecanismos que os indivíduos usam para decidir sobre quais atividades irão realizar e as viagens necessárias para a composição de sua agenda diária de atividades (Ettema, 1996).

No processo de simulação podem ser consideradas, em conjunto, as interações entre os membros do domicílio, o tipo de atividade que cada um deve realizar, os padrões de uso do solo e as restrições que cada membro do domicílio pode encontrar (como a estrutura familiar, os recursos financeiros disponíveis no domicílio, as restrições de tempo do indivíduo, o período de funcionamento dos estabelecimentos e a disponibilidade de automóvel) (Bhat e Lawton, 2000; Marshment, 2000, Mohammadian e Doherty, 2005; Goulias, 2005). Gliebe e Koppelman (2005) desenvolveram um estudo explorando o modo como os membros de um domicílio decidem incorporar atividades conjuntas e viagens compartilhadas nos seus padrões diários de atividades e viagens, considerando as restrições que podem ser encontradas em um domicílio. Este estudo revelou que características como as programações de trabalho, a presença de crianças no domicílio, as distâncias a serem percorridas e a disponibilidade de automóvel têm forte influência na programação de atividades conjuntas. Assim, estes fatores podem ser determinantes na opção do indivíduo por um ou outro modo de transporte, de acordo com as tarefas que ele precisa cumprir (Kitamura, 1996; Kurani e Lee-Gosselin, 1996).

Outro estudo considerando a interação entre os membros do domicílio foi desenvolvido por Pribyl e Goulias (2005). Nele os autores simularam o padrão de realização de viagens e atividades individuais e incorporaram as interações entre os membros do domicílio. O modelo utiliza ferramentas de simulação para extrair os padrões de atividades de um banco de dados e árvores de decisão para considerar as características pessoais e domiciliares.

Com ênfase dada principalmente à participação em atividades e focalizando na seqüência ou nos padrões de realização dessas atividades (utilizando como unidades de análise um longo período de tempo ou um dia completo), a abordagem baseada em atividades pode fornecer informações sobre o modo pelo qual os indivíduos e os domicílios modificam suas participações em atividades, em resposta à aplicação de determinadas políticas de transportes e de uso do solo, e como esse processo afeta a dinâmica urbana em uma cidade (Misra e Bhat, 2000).

De modo a evidenciar a necessidade do uso de uma abordagem baseada em atividades para o desenvolvimento de modelos mais sofisticados, é apresentada na Figura 1 a matriz evolutiva dos modelos de transpor- 
tes e uso do solo, na qual as linhas correspondem aos diferentes níveis de modelagem de uso do solo e as colunas aos níveis de modelos de demanda de transportes (Wegener e Fürst, 1999). De acordo com essa lógica, os modelos evoluem do canto superior esquerdo (L1/T1) para o canto inferior direito (L6/T4). Dessa forma fica fácil observar a transição da modelagem voltada apenas ao automóvel (T1) para a análise multimodal (T4).

Um processo de modelagem mais complexo, que admita a incorporação da inter-relação entre os sistemas de transportes e uso do solo e também as relações que ocorrem entre indivíduos em um domicílio ou no meio urbano, aponta a necessidade de desenvolvimento de modelos capazes de englobar o maior número possível de aspectos desses sistemas de forma clara, e que facilitasse o teste e a avaliação dos impactos da aplicação de políticas de transportes e uso do solo (Waddell e Ulfarsson, 2004) sobre a demanda de viagem. A busca de um modelo com estas características para o presente estudo exploratório levou à identificação e seleção do Albatross, cujos detalhes principais são apresentados no item contendo a metodologia.

\section{METODOLOGIA}

Com o intuito de atingir o objetivo proposto, o modelo Albatross foi implementado na cidade de São Carlos, no estado de São Paulo, considerada uma cidade de porte médio, com aproximadamente 200 mil habitantes à época da pesquisa (2004). Durante o processo de implementação foram identificadas três etapas fundamentais para avaliação de sua aplicação prática: i) a coleta dos dados necessários à calibração, ii) a sua adaptação às condições da cidade e iii) a análise dos resultados. Detalhes do modelo Albatross são apresentados no item 3.1, enquanto que os procedimentos de avaliação empregados nesse estudo exploratório são discutidos no item 3.2.

\subsection{O modelo Albatross}

A metodologia adotada para o desenvolvimento deste trabalho se baseia na utilização do modelo de atividades Albatross (Arentze e Timmermans, 2000), desenvolvido por pesquisadores da Universidade de Eindhoven, Holanda. É um modelo operacional, voltado a aplicações práticas, baseado em regras lógicas que representam heurísticas de escolha individuais obtidas a partir de um determinado conjunto de dados. $\mathrm{O}$ modelo incorpora grande parte dos conjuntos de escolha que envolve os padrões de atividades e apresenta um ambiente que possibilita a geração de cenários e ferramentas de avaliação.

No Albatross a demanda de viagem é considerada como derivada do processo pelo qual os indivíduos programam suas atividades e viagens em um período de tempo. As decisões são tomadas em nível domiciliar, sendo consideradas restrições espaço-temporais e institucionais no processo de programação das atividades diárias.

No modelo, as atividades são classificadas em fixas e flexíveis. Atividades fixas são aquelas consideradas obrigatórias, com local e horários predefinidos (como atividades de trabalho). Atividades flexíveis são aquelas cuja decisão de realização é tomada durante o dia, e não apresentam local nem horários fixos. O modelo considera que a geração de programações de atividades depende da natureza destas (fixas ou flexíveis) e da urgência em se realizar uma determinada atividade em um dia específico.

O processo de programação de atividades é conceitualizado como aquele no qual o indivíduo busca realizar objetivos particulares, dada uma variedade de restrições que limitam o número de padrões de atividades possíveis. Um padrão de atividades é a ordenação destas e suas respectivas viagens com local, tempo

\begin{tabular}{|c|c|c|c|c|c|}
\hline \multirow{2}{*}{\multicolumn{2}{|c|}{ Modelos de Transportes }} & T1 & $\mathrm{T} 2$ & T3 & \multirow{3}{*}{$\begin{array}{c}\text { T4 } \\
\text { Análise } \\
\text { multimodal, } \\
\text { baseada em } \\
\text { atividades }\end{array}$} \\
\hline & & $\begin{array}{l}\text { Não considera } \\
\text { transporte público e } \\
\text { escolha modal }\end{array}$ & $\begin{array}{c}\text { Considera o } \\
\text { transporte público, } \\
\text { não utiliza modelos } \\
\text { Logit, adota } \\
\text { período de análise } \\
\text { de } 24 \mathrm{~h} \\
\end{array}$ & $\begin{array}{l}\text { Considera o } \\
\text { transporte público, } \\
\text { uso de modelos } \\
\text { Logit, análise do } \\
\text { horário de pico }\end{array}$ & \\
\hline L1 & Nenhum & & & & \\
\hline L2 & Atividade & & & & \\
\hline L3 & $\begin{array}{l}\text { Alocação de usos do solo não } \\
\text { segue regras de mercado }\end{array}$ & & & & \\
\hline L4 & $\begin{array}{l}\text { Alocação tipo Logit com inclusão } \\
\text { de preços do solo urbano }\end{array}$ & & & & \\
\hline L5 & $\begin{array}{l}\text { Modelo do uso do solo de acordo } \\
\text { com regras de mercado }\end{array}$ & & & & \\
\hline L6 & $\begin{array}{l}\text { Modelo de uso do solo baseado } \\
\text { em atividades }\end{array}$ & & & & \\
\hline
\end{tabular}

Figura 1. Evolução da modelagem de transportes e uso do solo. Fonte: Wegener e Fürst (1999) 
de início, duração e modo de transporte definidos. Muitas vezes esse padrão é coordenado com padrões de atividades de outros indivíduos no domicílio (ou fora dele).

O uso do tempo e do espaço é condicionado pelos locais onde o individuo realiza suas atividades (denominados "bases”), sejam estes o domicílio, o local de trabalho etc. (Dijst e Vidakovic, 1997). O prisma espaço-tempo compreende o conjunto de posições no espaço e no tempo que o indivíduo pode visitar. De acordo com Dijst e Vidakovic (1997) a projeção do prisma no eixo do espaço fornece o espaço de ação potencial. Dessa forma, espaço de ação potencial é definido como a área contendo todos os locais de atividade que são alcançáveis, sujeito a um conjunto de condições espaço-temporais. Esse conjunto de condições inclui os tipos e as localizações das bases de atividades, intervalo de tempo disponível, velocidade de viagem e a proporção do tempo disponível gasto na viagem.

A programação de atividades individual é feita com base no contexto domiciliar. Decisões relacionadas aos modos de transporte definem o espaço de ação dentro do qual os indivíduos podem escolher as localizações para a realização de suas atividades. A organização das viagens em "elos” (viagens encadeadas) permite aos indivíduos realizar um maior número de atividades em um intervalo de tempo específico.

Identificadas as restrições que definem o espaço de ação dos indivíduos, a próxima etapa é a definição de como os indivíduos escolhem um entre os possíveis padrões de atividades. O modelo Albatross admite que os padrões de atividades irão ser construídos a partir de "mecanismos de aprendizado", onde o indivíduo determina um padrão de atividades de acordo com sua experiência (ou conhecimento). Esse padrão pode não ser o ótimo, uma vez que o indivíduo, baseado apenas em seus conhecimentos, pode desconsiderar outras opções viáveis.

O processo de aprendizagem transforma gradualmente padrões de busca em preferências individuais por determinadas localizações, tempo e modo de transporte para a realização de atividades. Essas preferências guiam o processo de tomada de decisão na construção de programações de atividades possíveis de serem realizadas. Rejeita-se então a noção de que indivíduos estão envolvidos em uma comparação sistemática de todos os possíveis padrões de atividades. O resultado desse aprendizado é um conjunto de regras que guiam o processo de solução do problema, o que permite aos indivíduos se adaptarem às condições de mudança.

Cabe salientar que o modelo Albatross se encontra em constante processo de atualização e melhoria, com a inserção de variáveis que possam fornecer resultados mais satisfatórios no processo de tomada de decisão. Por exemplo, pode-se citar o trabalho de Arentze e Timmermans (2005), no qual o custo de viagem é incorporado ao modelo. Outro aspecto que deve ser salientado, é que existem modelos baseados em atividades que usam em sua estrutura os princípios dos modelos de escolha discreta para determinação do comportamento de viagens e atividades individuais e domiciliares, como pode ser observado em Vovsha et al. (2004).

\subsection{Procedimentos de avaliação do estudo exploratório}

Os modelos de atividades possuem uma metodologia distinta para a obtenção dos dados necessários à sua calibração: são usados os diários de atividades em substituição aos diários de viagens (primeira etapa). Os diários de atividades são semelhantes aos diários tradicionais, mas requerem informações mais detalhadas sobre as atividades e viagens realizadas. Sua aplicação exige grande esforço para que os dados possam ser coletados, tanto na elaboração de sua estrutura como por parte dos entrevistadores (para maiores informações ver Arruda e Silva, 2004). Os dados foram coletados para uma amostra extraída de forma aleatória do cadastro de usuários do serviço de água e esgoto da cidade, abrangendo várias áreas da cidade, originalmente concebida para assegurar uma cobertura geográfica de toda a zona urbana, que na prática, no entanto, não chegou a se concretizar. Os entrevistados eram solicitados a preencher o diário de atividades por dois dias, entre terça-feira e quinta-feira (terça-feira e quarta-feira, terça-feira e quinta-feira ou quarta-feira e quinta-feira).

A adaptação do modelo compreendeu o ajuste de suas variáveis às condições encontradas no Brasil. Após identificadas as variáveis que necessitavam ser adaptadas, foi feito o ajuste das mesmas com base nos dados obtidos na etapa anterior. As adaptações necessárias foram realizadas a partir do uso de linguagem computacional, com mudanças no código fonte do modelo. No caso específico desta aplicação, as variáveis alteradas são descritas no item 4 deste artigo.

A última etapa desse processo foi a análise dos resultados obtidos com a calibração do modelo. Cabe ressaltar que esse processo não envolve, até o momento, a fase de validação do modelo, em função da quantidade limitada de dados coletados. Os resultados aqui apresentados são referentes apenas à fase de calibração do mesmo.

\section{RESULTADOS DA APLICAÇÃO DO MODELO}

Os principais resultados obtidos a partir da aplicação (que nesse caso resumem-se àqueles obtidos na fase 
de calibração) do modelo Albatross em uma cidade brasileira são apresentados e discutidos a seguir, com foco no processo de implementação do mesmo.

\subsection{Coleta de dados}

Os custos monetários associados a esta pesquisa foram da ordem de $\mathrm{R} \$ 18,00$ por domicílio entrevistado (cerca de seis dólares americanos, em janeiro de 2004), incluindo gastos com material necessário à coleta dos dados e com os pesquisadores. Neste último incluem-se gastos com o pagamento de horas trabalhadas ( $\mathrm{R} \$ 1,50$, ou cerca de US\$ 0,50 por hora) e os custos com transporte coletivo ( $\mathrm{R} \$ 2,30$, ou cerca de US\$ 0,76 por dia) para que estes pudessem se deslocar entre as áreas da cidade.

Como pode ser observado, o custo é relativamente baixo, sobretudo considerando-se a qualidade das informações que podem ser obtidas em relação às viagens e às atividades realizadas pelos indivíduos. Entretanto, uma das dificuldades encontradas foi o baixo nível de aceitação dos entrevistados em preencher o diário de atividades, o que comprometeu a quantidade dos dados coletados, conforme pode ser verificado na Tabela 1.

Tabela 1. Índice de aproveitamento do material de coleta de dados

\begin{tabular}{lr}
\hline Características do material & Quantidades \\
\hline Material distribuído & $343(100,00 \%)$ \\
Material válido & $155(45,19 \%)$ \\
Material não devolvido & $37(10,79 \%)$ \\
Material nulo & $96(27,99 \%)$ \\
Material em branco & $55(16,03 \%)$ \\
\hline
\end{tabular}

De $100 \%$ dos diários distribuídos, apenas cerca de $45 \%$ puderam ser usados na calibração do modelo. Cerca de $28 \%$ não puderam ser usados por estarem com informações incompletas ou pelo fato dos entrevistados terem preenchido apenas um dos dois dias solicitados. Cerca de $16 \%$ do material distribuído foi devolvido em branco e quase 11 \% não foi devolvido.

$\mathrm{Na}$ amostra, a divisão por gênero é equilibrada, sendo $55 \%$ do sexo masculino. Cerca de $15 \%$ dos domicílios têm presença de crianças - indivíduos com idade inferior a 12 anos de idade. A faixa etária predominante dos entrevistados se encontra entre 18 e 30 anos (63\%) e a grande maioria apresenta nível superior, variando de incompleto a pós-graduação (cerca de $90 \%)$. Os entrevistados possuem, na sua maioria, renda acima de $\mathrm{R} \$ 2.000,00$ por mês (47 \%) e a maior parte dos domicílios entrevistados possui pelo menos um automóvel (41 \%).

Cerca de $52 \%$ das viagens foram realizadas por automóvel (considerando a opção motorista e carona) e $39 \%$ foram realizadas pelo modo a pé. Os outros modos de transporte juntos (transporte coletivo, bicicleta e outros) somam apenas $9 \%$. Aproximadamente $42 \%$ das viagens até $1 \mathrm{~km}$ são realizadas por automóvel (como motorista ou passageiro) ou por transporte coletivo (3 \%). Em relação à participação em atividades, o modelo as classifica em "em casa” e "outro", significando as atividades que são realizadas fora do domicílio. Os dados mostram que $52 \%$ das atividades são realizadas fora do domicílio e que grande parte das atividades é por motivo "estudo" (28\%). O modelo não faz distinção entre as atividades realizadas no domicílio (restantes $48 \%$ ).

A pequena quantidade de diários efetivamente usados pode ter ocorrido em função de dificuldades encontradas pelos entrevistados em fornecer as informações requeridas. Cada entrevistado precisa descrever todas as atividades realizadas durantes dois dias úteis, bem como seus atributos (horários, local, modo de transporte utilizado etc.). A quantidade de informações é grande, pois quanto maior o numero de atividades que ele realiza, maior o número de informações que ele tem que descrever. Esse fato acaba por causar desinteresse por parte do entrevistado, ocasionando respostas incompletas ou a falta de informação. Outra observação feita foi a dificuldade dos entrevistados em compreender alguns termos usados e a ordem como as informações deveriam ser transcritas.

\section{2. $A$ adaptação do modelo}

As aplicações anteriores do Albatross demonstraram que os modelos baseados em atividades são capazes de fornecer informações detalhadas sobre a forma como as pessoas organizam suas atividades diárias, em função de um conjunto de características individuais, domiciliares e do ambiente urbano. No entanto, pelo fato do modelo usado ter sido desenvolvido originalmente na Holanda, alguns de seus parâmetros tiveram que ser adaptados para uso no contexto brasileiro.

O modelo original foi desenvolvido para detectar o comportamento de realização de atividade e viagens dos chefes dos domicílios (apenas cônjuges, não incluindo filhos). Nesta pesquisa, os dados foram coletados nos domicílios, qualquer que fosse sua composição. Foi solicitado que todos os membros do domicílio preenchessem o diário de atividades, mas isso não ocorreu em grande parte dos domicílios pesquisados. Assim, o modelo simulou o padrão de atividades e viagens das pessoas entrevistadas, não necessariamente os chefes dos domicílios, como em sua versão original. Como São Carlos se caracteriza como uma cidade universitária, grande parte dos domicílios entrevistados se constituíam nas chamadas "repúblicas" (pessoas, em geral estudantes sem grau de parentesco, morando em uma mesma residência). Nesse caso, o modelo original foi adaptado, e a variável posição no domicílio foi alterada em função dos dados obtidos. Assim, cada indivíduo entrevistado nas repúblicas foi considerado como residindo em domicílio composto 
por apenas um indivíduo. Uma vez que o modelo considera apenas as opções "trabalha" e "não trabalha", e não inclui a condição "estudante", todos os estudantes da amostra se encontram na categoria "não trabalha".

Em relação à disponibilidade de automóvel, adotouse a hipótese de que mesmo que existisse um veículo no domicílio, o indivíduo só foi considerado hábil para utilizá-lo se: possuísse carteira de habilitação e se tivesse realizado pelo menos uma viagem de automóvel como motorista com freqüência superior a duas vezes por semana. Esse critério pode ser verificado com o auxílio das outras perguntas feitas: freqüência de realização da atividade e se realizou a viagem como motorista ou passageiro.

Como o modelo considera o horário de funcionamento dos estabelecimentos comerciais para gerar padrões alternativos de realização de atividades, foi adotado o horário comercial de 8 horas da manhã até às 18 horas. Esses horários foram alterados em regiões com presença de grandes supermercados (das 8 às 20 horas) e do shopping center existente na cidade (das 10 às 22 horas).

O tempo de viagem foi estimado de acordo com a velocidade média dos modos de transporte nas vias. Para o automóvel $40 \mathrm{~km} / \mathrm{h}$, modo a pé $4,5 \mathrm{~km} / \mathrm{h}$, transporte público (ônibus) $18 \mathrm{~km} / \mathrm{h}$ e bicicleta $12 \mathrm{~km} / \mathrm{h}$. No caso do transporte público, onde para cada viagem de ônibus existem duas viagens a pé, foi considerado o ônibus como modo principal: ou seja, tanto a distância como o tempo dos trechos de caminhada nas pontas da viagem foram estimados como parte da viagem por ônibus. No caso do modo a pé e da bicicleta, no modelo Albatross eles são considerados como uma única categoria: modos lentos. No caso holandês, as viagens por bicicleta são predominantes, e sua velocidade foi aquela considerada no cálculo das distâncias das localidades possíveis de serem alcançadas. No caso de São Carlos, foram encontradas poucas viagens por bicicleta, o que tornou o modo a pé como dominante e a sua velocidade considerada para a iden- tificação dos locais de realização de atividades.

Em relação às informações de uso do solo, os dados holandeses, obtidos através de questionários enviados pelo correio, forneciam informações sobre o tipo de uso do solo predominante em cada região das localidades pesquisadas, estas delimitadas pela área coberta pelo código postal (no total, foram 23 regiões dispersas nas localidades pesquisadas). Assim, as atividades de compras, serviços e lazer poderiam ser realizadas apenas em locais específicos. No caso brasileiro, não foram obtidas informações sobre o uso do solo predominante. Dessa forma, adotou-se a hipótese, avaliada apenas de maneira informal, de que as pessoas poderiam realizar essas atividades em qualquer área da cidade, uma vez que vários estabelecimentos comerciais se encontram dispersos na cidade.

\subsection{Análise dos resultados}

Apesar das alterações discutidas no item 4.2, o modelo gerou resultados suficientes na fase de calibração para que alguns dos aspectos do padrão de comportamento de realização de atividades e viagens pudessem ser detectados, conforme pode ser observado nas Tabelas de 2 a 4 .

A Tabela 2 apresenta os resultados da freqüência das atividades realizadas fora de casa, quantificadas em relação à composição domiciliar, tanto os valores reais como aqueles obtidos com o modelo. Os valores em destaque nas Tabelas representam as porcentagens mais significativas. Algumas categorias de atividades são desagregadas como categorias distintas, como as atividades de lazer e visitas sociais, e atividades de compras diárias separadas de atividades de compras que não são realizadas diariamente.

Com base nos valores simulados, juntamente com as atividades de trabalho, as atividades de compras diárias, lazer e outras atividades não especificadas (categoria “Outras”) são as mais freqüentes no padrão observado. Essas mesmas atividades também são relevantes com base nos dados reais, acrescentando-se as

Tabela 2. Freqüência média (\%) das atividades individuais realizadas fora de casa com base na composição domiciliar (valores reais / valores simulados pelo modelo)

\begin{tabular}{|c|c|c|c|c|c|c|}
\hline \multirow{3}{*}{ Tipo de Atividade } & \multicolumn{6}{|c|}{ Composição Domiciliar } \\
\hline & \multicolumn{2}{|c|}{ Um indivíduo } & \multicolumn{3}{|c|}{ Mais de um indivíduo } & \multirow[b]{2}{*}{ TOTAL } \\
\hline & Não trabalha (\%) & Trabalha (\%) & $\begin{array}{c}\text { Nenhum trabalha } \\
(\%)\end{array}$ & $\begin{array}{l}\text { Apenas um } \\
\text { trabalha (\%) }\end{array}$ & $\begin{array}{l}\text { Mais de um } \\
\text { trabalha (\%) }\end{array}$ & \\
\hline Trabalho & $14,53 / 25,20$ & $1,99 / 2,00$ & 3,06 & $5,77 / 13,30$ & $7,55 / 15,4$ & $32,91 / 55,80$ \\
\hline Leva e traz & $0,50 / 0,70$ & $0,00 / 0,20$ & 1,14 & $1,28 / 0,10$ & $1,64 / 0,50$ & $4,56 / 1,50$ \\
\hline Não lazer & $4,27 / 1,70$ & $0,57 / 0,20$ & 1,00 & $1,70 / 2,20$ & $1,57 / 2,00$ & $9,19 / 6,10$ \\
\hline Compras diárias & 3,28 / 4,30 & $0,14 / 0,20$ & 1,64 & $1,71 / 2,90$ & $0,57 / 3,50$ & 7,34 / 10,90 \\
\hline Serviços & $0,00 / 0,60$ & $0,00 / 0,00$ & 0,00 & $0,00 / 0,50$ & $0,00 / 0,50$ & $0,00 / 1,50$ \\
\hline Compras não diárias & $0,07 / 0,50$ & $0,00 / 0,00$ & 0,00 & $0,00 / 0,90$ & $0,00 / 0,30$ & $0,07 / 1,70$ \\
\hline Visitas sociais & $0,14 / 1,40$ & $0,00 / 0,20$ & 0,07 & $0,00 / 0,30$ & $0,14 / 0,80$ & $0,36 / 2,80$ \\
\hline Lazer & $3,13 / 3,60$ & $0,00 / 0,20$ & 0,71 & $0,43 / 2,20$ & $1,28 / 1,70$ & $5,56 / 7,70$ \\
\hline Outras* & $16,67 / 7,60$ & $1,42 / 0,30$ & 5,48 & $7,34 / 2,00$ & $9,12 / 2,10$ & $40,03 / 12,00$ \\
\hline TOTAL & 42,59 / 45,50 & $4,13 / 3,50$ & 13,11 & 18,30 / 24,40 & 21,87 / 26,70 & $\begin{array}{c}100,00(\mathrm{n}=1404) / \\
100,00(\mathrm{n}=866)\end{array}$ \\
\hline
\end{tabular}

* Nesse caso, no banco de dados da amostra, a atividade "Volta para casa” foi incluída na categoria “Outras”. 
atividades de não lazer.

Em relação à composição domiciliar, um ponto deve ser destacado tanto nos dados reais como nos simulados, quando observados os valores relativos: a baixa freqüência de realização de atividades (mais especificamente a atividade trabalho, principal atividade fixa da programação diária individual) em domicílios com apenas um indivíduo e que trabalha e a alta freqüência desta atividade nessa mesma categoria de domicílio, mas em que ninguém trabalha. Por outro lado, quando observados os valores absolutos da simulação, observa-se que, na composição domiciliar com apenas um indivíduo que trabalha, do total de atividades realizadas (3,5\%), 2,0 \% corresponde a atividades de trabalho (cerca de 57,0 \% do total de atividades realizadas), o que é um valor significativo. Esse valor é muito próximo dos valores reais observados na amostra.

Já para o domicílio com apenas um indivíduo e que não trabalha, no que se refere aos valores simulados, do total de atividades realizadas (45,5 \%), 25,2 \% são atividades de trabalho (o que corresponde a 55,0 \% do total de atividades realizadas em termos absolutos). Já na amostra, os valores reais mostram que, para esta categoria, do total das atividades realizadas, cerca de 34,0 \% das atividades são de trabalho, considerandose os valores absolutos. Embora o resultado simulado seja aparentemente contrário à lógica, ele pode ser justificado pelo fato do modelo original considerar apenas as atividades de trabalho e não de estudo, conforme explicado anteriormente. Assim, a alta freqüência da atividade trabalho nos dados simulados pode não ser efetivamente uma indicação de atividades relacionadas ao trabalho, mas sim à atividade estudo, que ali se encontra incorporada.

Esse fato só poderia ter sido melhor esclarecido a partir de uma análise mais detalhada e com maior quantidade de informações e da possibilidade de alteração significativa do código fonte do programa que contém o modelo (o que não foi totalmente realizado no caso específico aqui relatado).

Um estudo mais detalhado também permitiria o esclarecimento do fato das previsões feitas pelo modelo para três tipos de atividades (leva e traz, não lazer e outras) terem apresentado grandes distorções quando comparadas em valores absolutos. O tipo de atividades "outras" inclui as atividades de volta para casa e assuntos pessoais.

O modelo não forneceu resultados em relação aos domicílios com mais de um indivíduo em que nenhum trabalha. Possivelmente, pelo fato do número de domicílios com essa característica ser muito pequeno, esses dados não foram considerados na simulação. Na grande maioria dos casos, esses domicílios eram compostos por aposentados cujo filho era estudante.

Apesar das ordens de grandeza entre os valores reais e os simulados serem diferentes, observa-se que, na maioria dos casos, os tipos de atividades mais relevantes em cada categoria de domicílio são os mesmos. Uma das mais expressivas diferenças encontra-se na categoria "Outras". Essa diferença pode ter sido causada pelo fato da atividade "Volta para casa" ter sido aí incluída na montagem do banco de dados da amostra, comportamento que acabou por não ser capturado pelo modelo. Vale observar que, por sugestão dos criadores do Albatross, a volta para casa foi considerada uma atividade, e não uma viagem. Para buscar melhor compreender a diferença nos resultados seria necessária uma investigação mais detalhada, seja nos dados, seja na estrutura do modelo.

A Tabela 3 mostra as freqüências das atividades realizadas fora de casa em relação à escolha do modo de transporte usado na viagem. Observa-se que a maior parte das viagens é realizada por automóvel, seguido pelos modos não motorizados, tanto nos dados reais como nos valores simulados. O automóvel predomina em grande parte das categorias de atividades, conforme pode ser observado nos valores em destaque. Os modos não motorizados são usados em cerca

Tabela 3. Freqüência (\%) do uso dos modos de transporte por tipo de atividade (valores reais/valores simulados pelo modelo) os valores em destaque representam os mais significativos em cada tipo de atividade

\begin{tabular}{|c|c|c|c|c|c|c|c|c|c|c|}
\hline \multirow[b]{2}{*}{$\begin{array}{l}\text { Modo de } \\
\text { transporte }\end{array}$} & \multicolumn{10}{|c|}{ Tipo de Atividade } \\
\hline & Trabalho & $\begin{array}{c}\text { Leva e } \\
\text { traz }\end{array}$ & $\begin{array}{l}\text { Não } \\
\text { lazer }\end{array}$ & $\begin{array}{l}\text { Compras } \\
\text { diárias }\end{array}$ & Serviços & $\begin{array}{l}\text { Compras } \\
\text { não } \\
\text { diárias }\end{array}$ & $\begin{array}{l}\text { Visitas } \\
\text { sociais }\end{array}$ & Lazer & Outras & TOTAL \\
\hline $\begin{array}{l}\text { Automóvel } \\
\text { (motorista) }\end{array}$ & $\begin{array}{c}14,35 / \\
25,20\end{array}$ & $\begin{array}{c}4,09 / \\
0,90\end{array}$ & $\begin{array}{c}3,95 / \\
4,60\end{array}$ & $\begin{array}{c}3,01 / \\
4,50\end{array}$ & $\begin{array}{c}0,00 / \\
0,30\end{array}$ & $\begin{array}{c}0,00 / \\
0,80\end{array}$ & $\begin{array}{c}0,14 / \\
1,20\end{array}$ & $\begin{array}{c}1,36 / \\
2,50\end{array}$ & $\begin{array}{c}17,14 / \\
5,50\end{array}$ & $44,05 / 45,50$ \\
\hline $\begin{array}{l}\text { Modos não } \\
\text { motorizados }\end{array}$ & $\begin{array}{c}13,92 / \\
16,90\end{array}$ & $\begin{array}{c}0,00 / \\
0,30\end{array}$ & $\begin{array}{c}0,22 / \\
0,80\end{array}$ & $\begin{array}{c}3,23 / \\
4,60\end{array}$ & $\begin{array}{c}0,00 / \\
0,80\end{array}$ & $\begin{array}{c}0,07 / \\
0,80\end{array}$ & $\begin{array}{c}0,14 / \\
1,00\end{array}$ & $\begin{array}{c}3,08 / \\
3,50\end{array}$ & $\begin{array}{c}14,92 / \\
4,40\end{array}$ & 35,58 / 33,10 \\
\hline $\begin{array}{l}\text { Transporte pú- } \\
\text { blico }\end{array}$ & $\begin{array}{c}3,37 / \\
5,60\end{array}$ & $\begin{array}{c}0,00 / \\
0,00\end{array}$ & $\begin{array}{c}0,36 / \\
0,20\end{array}$ & $\begin{array}{c}0,14 / \\
0,30\end{array}$ & $\begin{array}{c}0,00 / \\
0,10\end{array}$ & $\begin{array}{c}0,00 / \\
0,00\end{array}$ & $\begin{array}{c}0,00 / \\
0,10\end{array}$ & $\begin{array}{c}0,07 / \\
0,30\end{array}$ & $\begin{array}{c}2,80 / \\
0,20\end{array}$ & $6,74 / 6,80$ \\
\hline $\begin{array}{l}\text { Automóvel } \\
\text { (passageiro) }\end{array}$ & $\begin{array}{c}3,95 / \\
8,00\end{array}$ & $\begin{array}{c}0,43 / \\
0,20\end{array}$ & $\begin{array}{c}1,15 / \\
0,60\end{array}$ & $\begin{array}{c}0,86 / \\
1,50\end{array}$ & $\begin{array}{c}0,00 / \\
0,20\end{array}$ & $\begin{array}{c}0,00 / \\
0,10\end{array}$ & $\begin{array}{c}0,07 / \\
0,50\end{array}$ & $\begin{array}{c}1,08 / \\
1,50\end{array}$ & $\begin{array}{c}5,02 / \\
1,70\end{array}$ & $12,55 / 14,30$ \\
\hline Outros & $\begin{array}{c}0,50 / \\
0,20\end{array}$ & $\begin{array}{c}0,07 / \\
0,00 \\
\end{array}$ & $\begin{array}{c}0,07 / \\
0,00\end{array}$ & $\begin{array}{c}0,00 / \\
0,00\end{array}$ & $\begin{array}{c}0,00 / \\
0,00\end{array}$ & $\begin{array}{c}0,00 / \\
0,00\end{array}$ & $\begin{array}{c}0,00 / \\
0,00\end{array}$ & $\begin{array}{c}0,00 / \\
0,00\end{array}$ & $\begin{array}{c}0,43 / \\
0,10 \\
\end{array}$ & $1,08 / 0,30$ \\
\hline TOTAL & $\begin{array}{c}36,08 / \\
55,90\end{array}$ & $\begin{array}{c}4,59 / \\
1,40\end{array}$ & $\begin{array}{c}5,74 / \\
6,20\end{array}$ & $\begin{array}{l}7,25 / \\
10,90\end{array}$ & $\begin{array}{c}0,00 / \\
1,40\end{array}$ & $\begin{array}{c}0,07 / \\
1,70\end{array}$ & $\begin{array}{c}0,36 / \\
2,80\end{array}$ & $\begin{array}{c}5,60 / \\
7,80\end{array}$ & $\begin{array}{c}40,32 / \\
11,90\end{array}$ & $\begin{array}{c}100,00(\mathrm{n}=1404) \\
/ 100,00 \\
(\mathrm{n}=866)\end{array}$ \\
\hline
\end{tabular}


de $14 \%$ / $17 \%$ para a atividade trabalho e $3 \%$ / $5 \%$ para as atividades de compras (valores reais / valores simulados). Esses valores podem indicar a maior proximidade espacial entre locais de realização dessas atividades ao local da residência e/ou trabalho, possibilitando que as pessoas utilizem esse modo de transporte em parte de suas viagens. Uma interpretação ainda mais adequada dos resultados poderia ser obtida com maiores informações sobre a amostra, o que pode ser encontrado em Arruda (2005).

A Tabela 4 apresenta o resultado da árvore de decisão para a escolha do modo de transporte a ser usado para a atividade trabalho, gerada pelo algoritmo CHAID com base nos dados da amostra (Arentze e Timmermans, 2000). No Quadro 1 estão descritas as variáveis usadas. As primeiras linhas correspondem às variáveis que o algoritmo selecionou como as mais significativas e as linhas finais à probabilidade de escolha de cada modo de transporte. As colunas representam as partições feitas no conjunto de dados, pelo algoritmo CHAID. Para as variáveis independentes, estudam-se todas as partições e agrupamentos de categorias em sub-grupos, procurando aquele que tem o maior grau de significância com base em testes estatísticos. Uma nova análise é feita dentro de cada subgrupo resultante da partição anterior, estudando-se novamente a influência das variáveis independentes. Dessa forma tem-se uma escolha hierárquica de variáveis. O algoritmo realiza o processo até que seja obtida uma estrutura considerada aceitável e representativa dos dados.

Além dos resultados apresentados na Tabela 4, o algoritmo CHAID também foi aplicado para outras etapas do processo de decisão sobre quais atividades e viagens realizar. São elas: (a) a seleção das atividades flexíveis e da dimensão com quem a atividade será realizada, (b) a escolha da hora do dia em que as atividades serão realizadas, (c) as decisões de encadeamento das viagens e (e) a escolha do modo de transporte para cada encadeamento de viagem e da localização de realização da atividade. Os resultados obtidos para essas etapas não foram aqui descritos devido à limitação de espaço, mas podem ser encontrados em
Quadro 1. Descrição das variáveis usadas na escolha do modo de transporte para a atividade de trabalho

\begin{tabular}{|c|c|c|}
\hline Código & Descrição & Categoria \\
\hline Csec & $\begin{array}{l}\text { Classe socioeconômica do } \\
\text { domicílio }\end{array}$ & $\begin{array}{l}\text { 1: classe de renda baixa, } \\
2 \text { e 3: classe de valores } \\
\text { de renda média; } \\
\text { 4: classe de renda alta. }\end{array}$ \\
\hline Twork1 & $\begin{array}{l}\text { Tempo usado para a } \\
\text { realização da atividade de } \\
\text { trabalho/escola em análise } \\
\text { (min) }\end{array}$ & \multirow{3}{*}{$\begin{array}{l}\text { 1: tempos menores que } \\
240 \text { minutos; } \\
\text { 2: intervalos de tempo } \\
\text { entre } 241 \text { e } 360 \\
\text { minutos; } \\
\text { 3: intervalos de tempo } \\
\text { entre } 361 \text { e } 480 \\
\text { minutos; } \\
\text { 4: tempos maiores que } \\
480 \text { minutos. }\end{array}$} \\
\hline Ttot & $\begin{array}{l}\text { Tempo total usado na } \\
\text { realização de todas as ativida- } \\
\text { des realizadas fora do domicí- } \\
\text { lio (min) }\end{array}$ & \\
\hline Two & $\begin{array}{l}\text { Tempo total gasto em todas as } \\
\text { atividades de } \\
\text { trabalho/escola (min) }\end{array}$ & \\
\hline
\end{tabular}

Arruda (2005).

Os modos de transporte considerados como alternativa de escolha na árvore de decisão foram os modos não motorizados, o automóvel (passageiro e motorista) e o transporte público. Pelo fato da categoria "outros" (que inclui basicamente as viagens realizadas por motocicletas) ter apresentado uma porcentagem muito baixa na amostra pesquisada (0,5 \%), esta não foi considerada como uma opção válida neste caso.

De acordo com os resultados da Tabela 4, a primeira subdivisão se refere ao número de automóveis no domicílio. Na primeira combinação de variáveis (Nauto $=0$ e Csec $=1,2$ ) o resultado encontrado mostra que a escolha modal varia entre os modos não motorizados e o transporte público, que juntos atingem cerca de $52 \%$. Entretanto, o valor encontrado pelo modelo, com base nos dados usados em sua alimentação, para o modo automóvel, parece não ser consistente com as variáveis avaliadas (44\%), uma vez que indica um domicílio sem automóvel e de baixa classe socioeconômica. Na segunda combinação de variáveis (Nauto $=1$ ou mais e Csec $=1,2$ ), os resultados referentes à escolha do automóvel ainda parecem não estar apropriados. De acordo com a árvore de decisão, considerando um domicílio que possua pelo menos um automóvel e de classe econômica baixa, cerca de 46 \% dos indivíduos irão optar pelo modo automóvel (como

Tabela 4. Resultado da árvore de decisão para a escolha do modo de transporte para a atividade de trabalho

\begin{tabular}{|c|c|c|c|c|c|c|c|c|c|c|}
\hline \multirow{2}{*}{$\begin{array}{l}\text { Variáveis } \\
\text { Csec }\end{array}$} & \multicolumn{10}{|c|}{ Combinações de variáveis geradas na árvore de decisão } \\
\hline & $1-2$ & $1-2$ & $3-4$ & $3-4$ & $3-4$ & $3-4$ & $3-4$ & $3-4$ & $3-4$ & $3-4$ \\
\hline Genero & - & - & M & $\mathrm{F}$ & - & - & M & $\mathrm{F}$ & M & $\mathrm{F}$ \\
\hline Nauto & 0 & $\begin{array}{l}1 \text { ou } \\
\text { mais }\end{array}$ & - & - & - & - & - & - & - & - \\
\hline Twork1 & - & - & - & - & $1-3$ & 4 & - & - & - & - \\
\hline Ttot & - & - & $1-2$ & $1-2$ & 3 & 3 & 4 & 4 & - & - \\
\hline Two & - & - & $1-2$ & $1-2$ & $1-2$ & $1-2$ & $1-2$ & $1-2$ & $3-4$ & $3-4$ \\
\hline Modos de transporte & \multicolumn{10}{|c|}{ Probabilidades (\%) - os valores em destaque representam aqueles mais significativos } \\
\hline Modos não Motorizados & 25,9 & 14,6 & 33,3 & 47,6 & 34,0 & 10,3 & 11,1 & 9,1 & 60,0 & 60,0 \\
\hline Automóvel (motorista) & 44,4 & 19,5 & 33,3 & 23,8 & 47,2 & 89,7 & 57,8 & 84,8 & 40,0 & 10,0 \\
\hline Automóvel (passageiro) & 3,7 & 46,3 & 9,5 & 28,6 & 3,8 & 0,0 & 26,7 & 6,1 & 0,0 & 20,0 \\
\hline Transporte Público & 25,9 & 19,5 & 23,8 & 0,0 & 15,1 & 0,0 & 4,4 & 0,0 & 0,0 & 10,0 \\
\hline Total de domicílios & 27 & 41 & 21 & 21 & 53 & 29 & 45 & 33 & 30 & 20 \\
\hline
\end{tabular}


passageiro). Os resultados começam a representar de maneira mais adequada as escolhas individuais a partir do momento em que um conjunto maior de variáveis é avaliado. A subdivisão da variável gênero (Gênero = $\mathrm{M})$, em função tanto da classe econômica (Csec $=3,4)$ como do tempo usado na realização das atividades (Ttot e Two =1,2), mostra que a escolha modal é de cerca de 33\% para os modos não motorizados e automóvel (como) motorista e de $24 \%$ para o transporte coletivo.

Na subdivisão do Gênero $=\mathrm{F}$, com Csec $=3,4$, Ttot $=4$ e Two $=1,2$, a probabilidade de uso do automóvel é de cerca de 85 \%. Cabe ressaltar que, na subdivisão referente ao tempo usado para realização das atividades de trabalho (Twork1 = 4), considerando as características socioeconômicas (Csec $=3,4$ ), tempo total empregado na realização de todas as atividades (Ttot = 3), o modelo gerou uma combinação de variáveis inconsistente, atribuindo uma categoria de valores de tempo menor ao tempo gasto em todas as atividades realizadas e uma categoria maior ao tempo gasto na realização de uma atividade de trabalho específica, não sendo possível a interpretação de seus resultados. Essa aparente inconsistência do resultado gerado é um problema. Afirmar simplesmente que o problema está na base de dados não seria necessariamente correto, tampouco afirmar que o modelo não é capaz de captar as inconsistências. A melhor alternativa nesse caso seria usar a ferramenta Sylvia do modelo, destinada a verificar a consistência do banco de dados. Ela não foi usada no banco de dados considerado por motivos meramente operacionais, o que no entanto reforça a necessidade de estudar mais detalhadamente esse caso.

A aplicação dos modelos de atividades permite uma análise detalhada dos padrões de tomada de decisão individual e/ou domiciliar sobre quais atividades e viagens realizar. Entretanto, existe a necessidade que esses modelos sejam alimentados com boa quantidade de dados, que contenham informações tanto em relação às atividades e viagens realizadas, como também às características do ambiente urbano. Assim, ainda que os resultados aqui obtidos eventualmente não representem fielmente o comportamento de atividades e viagens dos indivíduos em geral, fornecem indicativos de que o modelo, se calibrado e validado com uma base robusta de dados, pode gerar resultados melhores que aqueles tradicionalmente usados. A principal diferença nesse caso, no entanto, seria a base conceitual subjacente ao modelo, que teoricamente tem melhores condições de reproduzir a realidade. Nesse sentido, a coleta dos dados por meio da aplicação de diários de atividades é a mais indicada, por fornecer grande parte das informações necessárias para a calibração do modelo, desde que tomados os cuidados acima mencio- nados.

\section{CONCLUSÕES}

Esta pesquisa teve como objetivo apresentar e discutir o potencial e as dificuldades de implementação de um modelo baseado em atividades em uma cidade brasileira. Esses modelos são muito usados em outros países, mas no Brasil não existe, até o momento, nenhum registro detalhado da aplicação dos mesmos. A análise do processo de aplicação do modelo de atividades foi realizada com foco em três aspectos: coleta de dados, adaptação do modelo Albatross às condições brasileiras e resultados obtidos na sua calibração. As principais conclusões, extraídas dos resultados apresentados, são discutidas na seqüência.

No que se refere ao primeiro aspecto analisado, a coleta de dados, a aplicação dos diários de atividades se apresentou viável, tanto em termos monetários como em qualidade das informações obtidas. Ficou claro, no entanto, que alguns procedimentos podem ser adotados para minimizar o número de informações inutilizadas. Por exemplo, o contato prévio com o entrevistado por telefone, antes do início da coleta, com o intuito de expor a necessidade de sua participação bem como o modo que os dados serão coletados (questionário e diário). Durante a pesquisa de campo, o entrevistado poderá ainda ser acompanhado pelo entrevistador (de modo a dirimir dúvidas e orientar no preenchimento dos diários). O tempo de aplicação dos diários deve ser estimado cuidadosamente, pois a quantidade de informações requeridas é grande. A estrutura do diário deve ser bem formulada, com a inclusão apenas de questões efetivamente relevantes e obedecendo a uma ordem lógica de preenchimento. Adicionalmente, a terminologia usada deve ser a mais clara possível, com caráter quase didático. Vale também coletar informações de atividades ocorridas dentro do domicílio (essas atividades estão sendo cada vez mais usadas na calibração de modelos de atividades).

A segunda etapa dessa análise, a adaptação, ainda que parcial, de um modelo estrangeiro às condições brasileiras mostrou-se possível, uma vez que esses são calibrados para a realidade do país em que foram desenvolvidos. No caso específico dos modelos de atividades, que englobam um grande número de variáveis em sua estrutura, esse processo fica mais difícil, se não se tem acesso pleno ao código fonte do modelo, dado que alguns dos parâmetros usados não são passíveis de modificações/alterações, o que compromete a qualidade do modelo e de sua aplicação.

Na terceira etapa do processo de aplicação do modelo, os resultados obtidos não permitiram uma análise conclusiva quanto aos padrões de ativida- 
des/viagens da amostra. A quantidade limitada de dados coletados permitiu que apenas a calibração do modelo fosse realizada. Dessa forma, a sua não validação impede uma avaliação plena do desempenho da implementação de um modelo baseado em atividades adaptado às condições brasileiras. A etapa de validação deverá ser realizada para que resultados conclusivos sejam alcançados. Ainda assim, pode-se concluir, com base no estudo exploratório aqui relatado, que o desenvolvimento de um modelo de atividades por pesquisadores brasileiros é perfeitamente viável, uma vez que estes possuem toda a infra-estrutura necessária, tanto em termos de equipamentos como de mão de obra especializada. Nesse sentido, pesquisas mais recentes, que se fundamentam fortemente na teoria subjacente aos modelos baseados em atividades, como os trabalhos de Silva (2006), Silva e Kawamoto (2006) e Pitombo (2007), constituem importantes passos nessa direção.

Adicionalmente, durante a execução desta pesquisa verificou-se que os modelos baseados em atividades possuem uma estrutura complexa, por incorporarem muitos aspectos relacionados às atividades (horários de início e término, localização etc), às viagens (modo de transporte disponível, tempo de viagem, horários de início e término) e aos indivíduos (composição domiciliar, restrições encontradas em função da organização domiciliar etc). O tratamento e manipulação dessas informações exige o uso de técnicas de programação e análises estatísticas, o que também não se constitui em uma barreira para sua implantação no Brasil, dado que são áreas bem desenvolvidas no país.

\section{AGRADECIMENTOS}

Os autores agradecem ao CNPq (Conselho Nacional de Desenvolvimento Científico e Tecnológico), à CAPES (Fundação Coordenação de Aperfeiçoamento de Pessoal de Nível Superior) e à FAPESP (Fundação de Amparo à Pesquisa do Estado de São Paulo) por contribuírem para diferentes fases do desenvolvimento da pesquisa relativa aos modelos baseados em atividades que deu origem a esse artigo. Reconhecem e agradecem também aos revisores anônimos da revista Transportes pelas excelentes sugestões e comentários.

\section{REFERÊNCIAS BIBLIOGRÁFICAS}

Arentze, T.; Timmermans, H (2000). Albatross - A learning based transportation oriented simulation system. Technische Universiteit Eindhoven - European Institute of Retailing and Services Studies. $518 \mathrm{p}$.

Arentze, T.; Timmermans, H. (2005) Parametric Action Decision Trees: Incorporating Continuous Attribute Variables into Rule-Based Models of Activity-Travel Behavior. In: Annual Transportation Research Board Meeting, 84 ${ }^{\text {th }}, 2005$, Washington, D.C. Proceedings... Washington, D.C.: Transportation Research Board. CDROM.

Arruda, F. S.; Silva, A. N. R. (2004) Diários de atividades: uma metodologia alternativa de coleta de dados para planejamento de transportes. In: Panorama Nacional da Pesquisa em Transportes 2004, vol I. Associação Nacional de Pesquisa e Ensino em Transportes, pp. 675-686.

Arruda, F.S. (2005) Aplicação de um modelo baseado em atividades para análise da relação uso do solo e transportes no contexto brasilei- ro. Tese (Doutorado). Escola de Engenharia de São Carlos, Universidade de São Paulo, São Carlos.

Bhat, C.; Lawton, K. (2000) Passenger travel demand forecasting. In: Annual Transportation Research Board Meeting, $79^{\text {th, }}$ 2000, Washington, D.C. Proceedings... Washington, D.C.: Transportation Research Board. CD-ROM.

Chapin, F. S. (1971) Free-time activities and the quality of urban life. Journal of the American Institute of Planners. v. 37, p. 411-417.

Cullen, I.; Godson, V. (1975) Urban networks: the structure of activities patterns. Progress in Planning. n. 4, p.1-96.

Dijst, M.; Vidakovic, V. (1997) Individual actions space in the city. In: Ettema, D. F.; Timmermans, H. J. P. (Ed.). Activity-based approaches to travel analysis. p. 117-113.

Ettema, D. (1996) Activity-based travel demand modeling. Eindhoven. 280 f. Tese (Doutorado em Planejamento Urbano), Universidade de Eindhoven, Holanda.

Gliebe, J. P.; Koppelman, F. S. (2005) Modeling Household ActivityTravel Interactions as Parallel Constrained Choices. In: Annual Transportation Research Board Meeting, 84 ${ }^{\text {th }}, 2005$, Washington, D.C. Proceedings... Washington, D.C.: Transportation Research Board. CD-ROM.

Goulias, K. (2005) An Analysis of Activity Type Classification and Issues Related to the With Whom and For Whom- Questions of an Activity Diary. In: Annual Transportation Research Board Meeting, $84^{\text {th }}$, 2005, Washington, D.C. Proceedings... Washington, D.C.: Transportation Research Board. CD-ROM.

Hagerstrand, T. (1970) What about people in regional science? Papers and Proceedings of the Regional Science Association. n. 24, pp. 7-24.

Joh, C.H.; Huh, W. (2005) Activity-Travel Behavior in Time and Space: An analysis of commuters' weekday trips involving convenience goods purchase in Seoul, Korea. In: Annual Transportation Research Board Meeting, 84 ${ }^{\text {th }}$, 2005, Washington, D.C. Proceedings... Washington, D.C.: Transportation Research Board. CDROM.

Kitamura, R. (1996) Activity-based travel forecasting: what are some issues? Applications of models of activity behavior for activity based demand forecasting. In: Activity-Based Travel Forecasting Conference, 1996. Proceedings... Disponível em: $<$ http://www.bts.gov/tmip/papers/tmip/abtf/kitamura.htm>. Acesso em 22 de outubro de 1998.

Kurani, K.; Lee-Gosselin, M. E. H. (1996) Synthesis of past activity analysis applications. In: Activity-Based Travel Forecasting Conference. Proceedings.... Disponível em: <http://www.bts.gov/ tmip/papers/tmip/abtf/kurani.htm>. Acesso em 22 de outubro de 1998.

Marshment, R. (2000) Transportation planning challenges and opportunities. In: Annual Transportation Research Board Meeting, $79^{\text {th }}$, 2000, Washington, D.C. Proceedings... Washington, D.C.: Transportation Research Board. CD-ROM.

Misra, R.; Bhat, C. (2000) Activity-travel patterns of non-workers in the San Francisco Bay Area: an exploratory analysis. In: Annual Transportation Research Board Meeting, $79^{\text {th }}$, 2000, Washington, D.C. Proceedings... Washington, D.C.: Transportation Research Board. CD-ROM.

Mohammadian, A.; Doherty, S. (2005) A Mixed Logit Model of Activity Scheduling Time Horizon Incorporating Spatial-Temporal Flexibility Variables. In: Annual Transportation Research Board Meeting, $84^{\text {th }}$, 2005, Washington, D.C. Proceedings... Washington, D.C.: Transportation Research Board. CD-ROM.

Pendyala, R. M.; Kitamura, R.; Reddy, D.V.G.P. (1998) Application of an activity-based travel demand model incorporating a rule-based algorithm. Environment and Planning B. v.25, p. 753-772.

Pitombo, C. S. (2007) Estudos de relações entre variáveis socioeconômicas, de uso do solo, participação em atividades e padrões de viagens encadeadas urbanas. Tese (Doutorado). Escola de Engenharia de São Carlos, Universidade de São Paulo, São Carlos.

Pribyl, O.; Goulias, K. G. (2005) Simulation of daily activity patterns incorporating interactions within households: Algorithm overview and performance. In: Annual Transportation Research Board Meeting, $84^{\text {th }}$, 2005, Washington, D.C. Proceedings... Washington, D.C.: Transportation Research Board. CD-ROM.

Silva, M. A. (2006) Verificação da aplicabilidade da técnica de mineração de dados na previsão da demanda por transporte de passageiros urbanos usando dados da Região Metropolitana de São Paulo. Dissertação (Mestrado). Escola de Engenharia de São Carlos, Universidade de São Paulo, São Carlos. 
Silva, M. A.; Kawamoto, E. (2006) Aplicação da técnica de mineração de dados na previsão da demanda por passageiros urbanos. In: $\mathrm{Pa}$ norama Nacional da Pesquisa em Transportes 2006, vol. I. Associação Nacional de Pesquisa e Ensino em Transportes, pp. 528540 .

Vovsha, P.; Bradley, M.; Bowman, J. L. (2004) Activity-based travel forecasting models in the United States: Progress since 1995 and Prospects for the Future. In: EIRASS Conference on Progress in Activity-Based Analysis, Vaeshartelt Castle, Maastricht, The Netherlands. Disponivel em: <http://jbowman.net $>$.

Waddell, P.; Ulfarsson, G. F. (2004) Introduction to urban simulation: Design and development of operational models. In: P. Stopher, K. Button, K. Kingsley, e D. Hensher (editors.) Handbook in Transport, volume 5: Transport Geography and Spatial Systems, pp. 204-236. Pergamon Press, Elmsford, NY, USA. Disponível em: $<$ http://www.urbansim.org.>.

Wegener, M.; Fürst, F. (1999) Land-use transport interaction: state of the art. Publicação 46 (Berichte aus dem Institut für Raumplanung 46). Institut für Raumplanung, Universität Dortmund, Dortmund. Disponível em: <http://www.inro.tro.nl/ transland/Deliverable\%202a.pdf>. 